\title{
Associations of hand washing frequency with the incidence of illness: a systematic review and meta-analysis
}

\author{
Yangqin Xun ${ }^{1,2,3,4} \wedge$, Qingxia Shi ${ }^{5,6}$, Nan Yang ${ }^{5,6}$, Nan Yang ${ }^{1,2,3,4}$, Yan $\mathrm{Li}^{7}$, Wenwen $\mathrm{Si}^{7}$, Qianling Shi \\ Zijun Wang ${ }^{1,2,3,4}$, Xia Liu ${ }^{7}$, Xuan Yu ${ }^{1,2,3,4}$, Qi Zhou ${ }^{8}$, Minyan Yang ${ }^{7}$, Yaolong Chen ${ }^{1,2,3,4}$
}

${ }^{1}$ Evidence-Based Medicine Center, School of Basic Medical Sciences, Lanzhou University, Lanzhou, China; ${ }^{2}$ An Affiliate of the Cochrane China Network, Lanzhou University, Lanzhou, China; ${ }^{3}$ World Health Organization (WHO) Collaborating Centre for Guideline Implementation and Knowledge Translation, Lanzhou, China; ${ }^{4}$ Lanzhou University GRADE Center, Lanzhou, China; ${ }^{5}$ Department of Respiratory, Children's Hospital of Chongqing Medical University, National Clinical Research Center for Child Health and Disorders, Ministry of Education Key Laboratory of Child Development and Disorders, Chongqing, China; ${ }^{6}$ Chongqing Key Laboratory of Pediatrics, Chongqing, China; ${ }^{7}$ School of Public Health, Lanzhou University, Lanzhou, China; ${ }^{8}$ The First School of Clinical Medicine, Lanzhou University, Lanzhou, China

Contributions: (I) Conception and design: Y Xun, Y Chen; (II) Administrative support: Y Chen; (III) Provision of study materials or patients: Y Xun, Q Shi, N Yang, N Yang, M Yang; (IV) Collection and assembly of data: Q Shi, Z Wang, X Yu, Q Zhou, X Liu; (V) Data analysis and interpretation: Y Xun, Y Li, W Si; (VI) Manuscript writing: All authors; (VII) Final approval of manuscript: All authors.

Correspondence to: Yaolong Chen. Evidence-based Medicine Center, School of Basic Medical Sciences, Lanzhou University, Lanzhou 730000, China. Email: chenyaolong@lzu.edu.cn.

Background: Hand hygiene is one of the ways to prevent the spread of diseases. Our aim was to explore the relationship between hand washing frequency and the impact on disease, and give recommendations on the number of times to wash hands.

Methods: We searched seven electronic databases from their inception to April 11, 2020, and reference lists of related reviews for all studies on hand washing frequency and disease prevention. The Review Manager 5.3. software was used to conduct a meta-analysis. We assessed the risk of bias of included studies, and quality of evidence of the main findings.

Results: A total of eight studies were included. The results of the meta-analysis showed that there was no statistical significance between the effect of disease prevention and washing more than 4 times/day compared to not [odds ratio $(\mathrm{OR})=0.61,95 \%$ confidence interval $(\mathrm{CI}): 0.37$ to 1.01 ]. The results of a case-control study showed that compared with hand washing $\leq 4$ times/day, hand washing 5-10 times/day (OR =0.75, 95\% CI: 0.63 to 0.91 ) and hand washing $>10$ times/day ( $\mathrm{OR}=0.65,95 \%$ CI 0.53 to 0.80 ) could reduce the risk of disease infection. There was no statistical significance advantage to hand washing more than 10 times/day compared to $5-10$ times/day ( $\mathrm{OR}=0.86,95 \% \mathrm{CI}$ : 0.70 to 1.06$)$. Comparing hand washing $\leq 10$ times/day with hand washing $>10$ times/day, increased hand washing was a protective factor against infection $(\mathrm{OR}=0.59,95 \% \mathrm{CI}$ : 0.36 to 0.97$)$.

Conclusions: The more frequently hands were washed, the lower risk of disease. So far however, there is no high-quality evidence indicating the best range of hand washing frequency for disease prevention.

Keywords: Hand washing; diseases prevention; meta-analysis; systematic review

Submitted Aug 20, 2020. Accepted for publication Dec 11, 2020.

doi: $10.21037 /$ atm-20-6005

View this article at: http://dx.doi.org/10.21037/atm-20-6005

$\wedge$ ORCID: 0000-0002-5190-606X. 


\section{Introduction}

Dirty hands can be a vector for several gastrointestinal infections, such as diarrhea (1), and respiratory infections, such as influenza, severe acute respiratory syndrome (SARS), and coronavirus disease 2019 (COVID-19) (2-5). These infectious diseases can cause serious complications, especially for people with poor compromised immunity (6-8). Diarrhea and pneumonia, for example, are the top two leading causes of mortality among children under five years of age, causing approximately 1.8 million deaths each year (9). Since December 2016, the main cause of excess all-cause mortality in many European countries, especially among people over 65 years of age, has been the influenza virus (10). Subsequently, hand hygiene is one of the most effective ways to prevent the spread of these infectious diseases and reduce associated morbidity and mortality (11).

Numerous studies have shown that proper hand washing can prevent the transmission of certain diseases (12-15). Specifically, promoting the frequency of hand washing can prevent about $30 \%$ of diarrhea-related illnesses (16) and about $20 \%$ of respiratory infections (17). Through efforts such as community hand washing education, diarrhea can be reduced by $23-40 \%$ (17-19). For people with poor compromised immunity, their proper hand washing can reduce certain infectious diseases by $58 \%$ (20), while respiratory diseases such as cold will be reduced by $16-21 \%(17,18,21)$. This simple prevention measure can also have lasting impacts. Some research has reported that rates of absenteeism among primary school students due to gastrointestinal diseases can be reduced by $29-57 \%$ with the implementation of hand washing (22). For this reason, The World Health Organization (WHO) advises the public to wash their hands frequently as a part of basic protection against COVID-19 (23).

While some studies have shown that increasing the frequency of hand washing can effectively prevent illness, there is no advice regarding the specific frequency of washing hands that is needed. A study by Alba et al. showed that for every unit of improvement in hand washing frequency, typhoid infection rates decreased by about $62 \%$ (24). The study found that hand washing more than 4 times per day was a protective factor in reducing disease risk (25). However, some studies have reported no statistical difference in the prevention of diseases due to the frequency of hand washing $(26,27)$. Thus, research on the frequency of hand washing and its effect on prevention of disease is not consistent. Hence, the purpose of this study was to conduct a systematic review to explore the relationships between the frequency of hand washing and the effect of disease prevention, and develop more comprehensive suggestions on the frequency of hand washing. We present the following article in accordance with the PRISMA reporting checklist (available at http:// dx.doi.org/10.21037/atm-20-6005).

\section{Methods}

We pre-registered our meta-analysis at PROSPERO (CRD42020183002).

\section{Search strategy}

We searched the following electronic databases from their inception to April 11, 2020: Cochrane library, MEDLINE (via PubMed), Embase, Web of Science, China Biology Medicine disc (CBM), China National Knowledge Infrastructure (CNKI), and Wanfang Data. The WHO Clinical Trials Registry Platform, US National Institutes of Health Trials Register, the International Standard Randomized Controlled Trial Number [ISRCTN] Register, the official websites of the WHO and Centers for Disease Control (CDC), Google Scholar (first 100 records), and reference lists of relevant reviews were also scanned. Mesh terms included prevent*, safeguard, precaution, defense, prophylaxis, "prevention and control", "preventive therapy", "preventive measures", handwashing*, "hand washing*", hand-washing, hand-cleaning, "hand hygiene", "wash* hand*", "clean hand"”, frequency, times, seldom, always, and often. A full list of search terms can be found in Appendix 1.

\section{Eligibility criteria}

A number of inclusion criteria were considered, including (I) randomized controlled trial (RCT), cohort study, casecontrol study, cross-sectional study; (II) participants were not limited by age, gender, race, or type of disease; (III) studies on hand washing frequency of participants and effect on disease prevention of participants. Studies were excluded based on the following criteria: (I) no clear frequency of hand washing; (II) duplicate studies; or (III) articles were written in languages other than English or Chinese; or (IV) abstracts, comment letters, or reviews.

The primary interest was the impact of frequency of hand washing on disease risk (defined as disease infection 
risk, reinfection risk, or episodes risk). The secondary outcome of interest was the symptoms of the disease, or adverse effects.

\section{Study selection}

After removing those studies which were duplicates, two researchers independently selected the studies using Endnote. The first stage of screening was based on article titles and abstracts. Second, for articles with incomplete information and ambiguous titles, the full-text was downloaded and reviewed in order to determine inclusion. Inconsistent results were resolved through discussion or a third researcher was consulted if necessary. The detailed literature screening process is shown in Figure 1.

\section{Data extraction}

Two researchers extracted data independently with a predesigned data collection form, which was reviewed, and disagreements were resolved by consensus or consulting a third researcher. Information extracted from the studies included: (I) basic information (title, first author, publication year, type of study, single-center or multi-center study, sample size, and disease); (II) participants (age and gender); (III) intervention (specific hand washing frequency); and (IV) results (the total number, mean, and standard deviation of disease cases or symptom occurrence).

\section{Assessment of risk of bias}

Two researchers independently assessed the potential bias in each included study. Discrepancies were resolved by discussion or with consensus from a third researcher. RCTs were assessed for bias according to the Cochrane risk-ofbias tool ( $\mathrm{RoB})$ (28) consisting of seven domains: random sequence generation, allocation concealment, blinding of participants and personnel, blinding of outcome assessment, incomplete outcome data, selective outcome reporting, and other bias. Each potential source of bias as was graded "Low", "Unclear" or "High". High risk of bias means the study is judged to be at high risk of bias in at least one domain for this result, or the study is judged to have some concerns for multiple domains in a way that substantially lowers confidence in the result. Cohort and case-control studies were assessed for the risk of design-specific bias using the relevant Newcastle-Ottawa Scale (NOS) (29), which consists of three aspects: the selection of exposure, comparability, and assessment of outcome. The maximum score was nine, and scores of seven or more were graded as high quality while scores of less than seven were defined as low quality. For cross-sectional studies, the Agency for Healthcare Research and Quality Scale (AHQR) (30) was used to assess for bias. It consists of 11 items, that are answered with "Yes", "No" or "Unclear".

\section{Statistical analysis}

A meta-analysis of outcomes for the compatible data was conducted using Review Manager 5.3 software (a software was recommended by Cochrane Handbook for developing systematic reviews) (31), which means the frequency of hand washing included in the same range can be conducted metaanalysis. A descriptive analysis of outcome indicators that could not be combined quantitatively was also conducted. Meta-analyses of binary variables were performed using the Mantel-Haenszel method and continuous variables using the inverse variance method. Dichotomous data were evaluated using odds ratio (OR) or risk ratio (RR) with $95 \%$ confidence intervals (CI). Continuous data were analyzed using the weighted mean difference (WMD) with 95\% CI. Two-sided $\mathrm{P}$ values of $<0.05$ were considered statistically significant. We assessed evidence of heterogeneity using the $\chi^{2}$ test and $\mathrm{I}^{2}$ statistic. $\mathrm{P}<0.10$ was considered to be consistent with statistically significant heterogeneity. A random-effects model was used for $\mathrm{I}^{2}>50 \%$ and a fixed effect model was used for $\mathrm{I}^{2} \leq 50 \%$. We conducted a subgroup analysis according to the study design to explore the reasons for substantial heterogeneity. Publication bias was assessed by the Egger test.

\section{Quality of evidence assessment}

Two reviewers assessed the quality of main evidence independently using the Grading of Recommendations Assessment, Development and Evaluation (GRADE) tool. A "Summary of Findings" table using the GRADEpro software (32) was created, which shows the overall grading of the body of evidence for each pre-specified outcome that was accounted for in the meta-analysis. Direct evidence from RCTs starts at high quality, and evidence from observational studies at low quality. The quality can be downgraded for five different reasons (study limitations, consistency of effect, imprecision, indirectness, and publication bias) and upgraded for three reasons (large magnitude of effect, dose-response relation, and plausible 


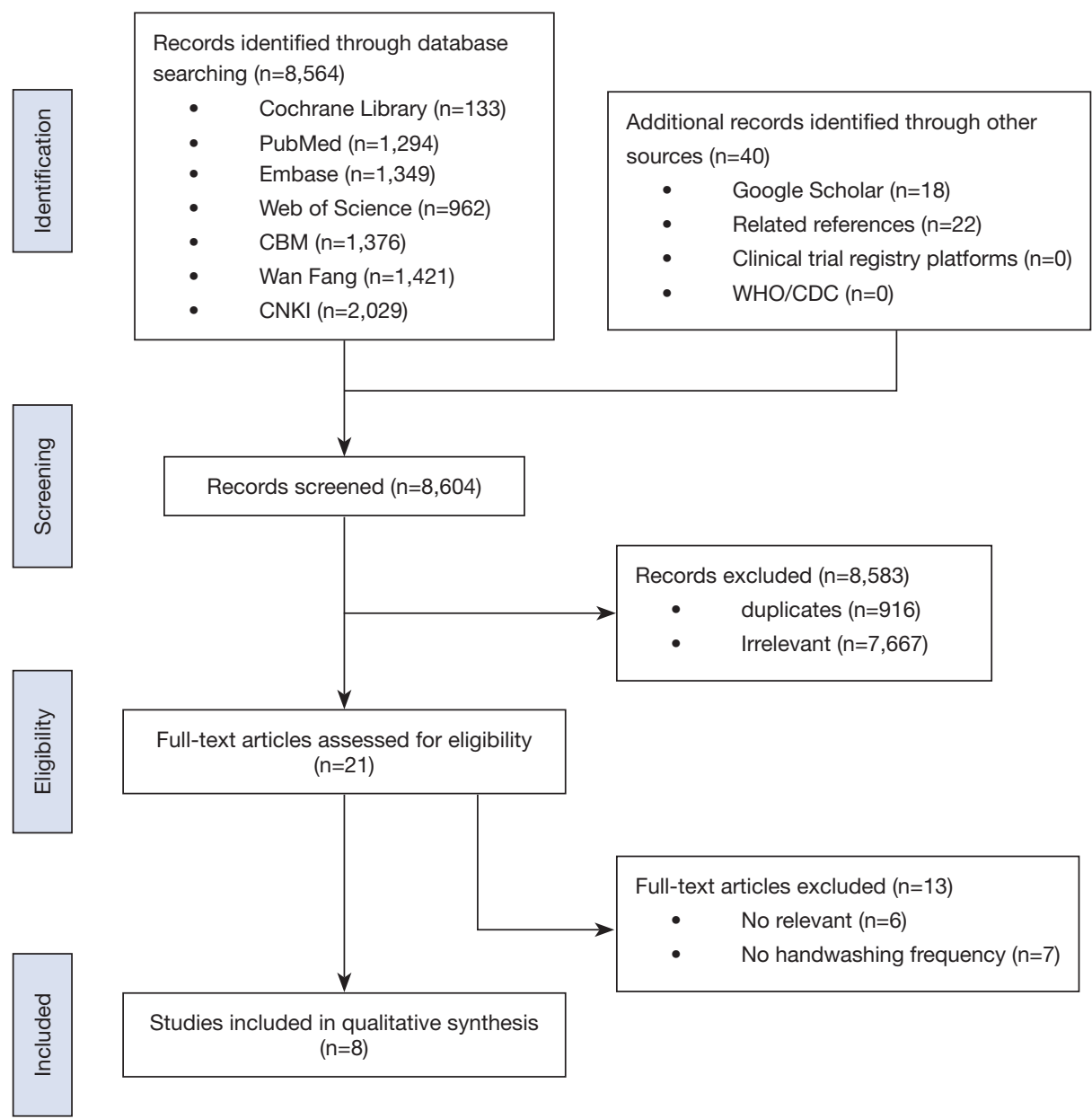

Figure 1 Flow diagram of the study selection process.

confounders or biases) (33-36). The quality of evidence for each outcome is classified as "high", "moderate", "low", or "very low" reflecting to what extent we can be confident that the effect estimates are correct.

\section{Results}

\section{Study selection and characteristics}

Figure 1 shows the literature retrieval and screening process. We identified 8,604 studies, 916 duplicated studies and 7,667 irrelevant studies were excluded, leaving 21 potentially eligible studies to be reviewed. After reading the full-text, six studies with no relevant outcome indicators and seven studies that did not report hand-washing frequency were excluded. Finally, a total of eight studies were included in the meta-analysis $(14,15,20,25-27,37,38)$. Table 1 shows the included study characteristics of two RCTs $(20,27)$, one cohort study (37), four case-control studies $(14,15,25,26)$, and one cross-sectional study (38). Four studies (14,25-27) used hand washing 4 times per day as the cut-off value and two studies $(14,15)$ used hand washing 10 times per day as the cut-off value. The study population in five of the studies was patients $(14,15,20,25,26)$. The basic characteristics of included studies and baseline characteristics of participants of each included study is summarized in Tables 1 and 2 .

The quality of included studies was assessed using the ROB, NOS and AHRQ (see Tables S1-S3). Overall, two and three items of the two RCTs were evaluated as "Low", while the rest were "Unclear". Two of the case-control studies were graded as eight stars, while two were seven stars, and one cohort study was also seven stars. Six items from the cross-sectional study were evaluated as "Yes", two items were "Unclear", and three items were classified 
Table 1 Basic characteristics of the included studies

\begin{tabular}{|c|c|c|c|c|c|c|}
\hline $\begin{array}{l}\text { Study } \\
\text { (publication year) }\end{array}$ & Study design & Country & $\begin{array}{l}\text { Single/multi-center } \\
\text { study }\end{array}$ & Sample & $\begin{array}{l}\text { Hand washing } \\
\text { frequency }\end{array}$ & Disease \\
\hline Godoy 2012, (14) & Case-control & Spain & Multi-center & 3,087 & $\begin{array}{l}1-4,5-10 \\
>10 \text { times/day }\end{array}$ & Influenza A \\
\hline Lau 2004, (15) & Case-control & China & Single-center & 990 & $1-10,>10$ times/day & $\begin{array}{l}\text { Acute respiratory tract } \\
\text { infection, influenza-like illness }\end{array}$ \\
\hline Huang 2007, (20) & RCT & America & Single-center & 148 & 4, 7 times/day & Diarrhea \\
\hline $\begin{array}{l}\text { Mangklakeree } \\
2014,(27)\end{array}$ & $\mathrm{RCT}$ & Thailand & Single-center & 454 & $\leq 3,>3$ times/day & Influenza-like illness \\
\hline Chen 2011, (25) & Case-control & China & Single-center & 132 & $<4, \geq 4$ times/day & Influenza A \\
\hline
\end{tabular}

$\mathrm{RCT}$, randomized controlled trial; SARS, severe acute respiratory syndrome.

Table 2 Baseline characteristics of participants

\begin{tabular}{|c|c|c|c|c|c|}
\hline Study & Participants & Gender (male/female) & $\begin{array}{l}\text { Age of test/control (years), } \\
\text { (range or mean } \pm \mathrm{SD} \text { ) }\end{array}$ & Test group (n) & Control group (n) \\
\hline Godoy 2012, (14) & $\begin{array}{l}\text { Patients and matching } \\
\text { controls }\end{array}$ & $1,507 / 1,580$ & $38.48 \pm 22.79 / 39.13 \pm 22.68$ & 813 & 2,274 \\
\hline Lau 2004, (15) & $\begin{array}{l}\text { Patients and matching } \\
\text { controls }\end{array}$ & $475 / 515$ & $\geq 16$ & 330 & 660 \\
\hline Huang 2007, (20) & AIDS patients & $111 / 37$ & $43.70 \pm 11.00 / 41.90 \pm 11.00$ & 75 & 73 \\
\hline Mangklakeree 2014, (27) & Elementary school student & $216 / 238$ & - & 175 & 279 \\
\hline Torner 2015, (26) & $\begin{array}{l}\text { Patients and matching } \\
\text { controls }\end{array}$ & $269 / 209$ & $5.40 \pm 4.50 / 5.30 \pm 4.60$ & 239 & 239 \\
\hline
\end{tabular}

SD, standard deviation; AIDS, acquired immune deficiency syndrome; -, unavailable.

as "No". Table S4 provides a summary of the quality of evidence for each outcome.

\section{The results of hand washing frequency and disease prevention $>4$ times per day versus $\leq 4$ times per day}

Four studies $(14,25-27)$ specifically considered the impact of washing your hands less than 4 times per day $v$ s. washing them more than 4 times per day (Figure 2). Heterogeneity test showed $\mathrm{I}^{2}=85 \%$, so the random-effects model was used. The meta-analysis results found no statistical significance of washing your hands more than 4 times or less than 4 times a day and respiratory disease prevention $(\mathrm{OR}=0.61,95 \%$ CI: 0.37 to 1.01$)$. When we performed a subgroup analysis by study design, a meta-analysis of three case-control studies $(14,25,26)$ showed that hand washing more than 


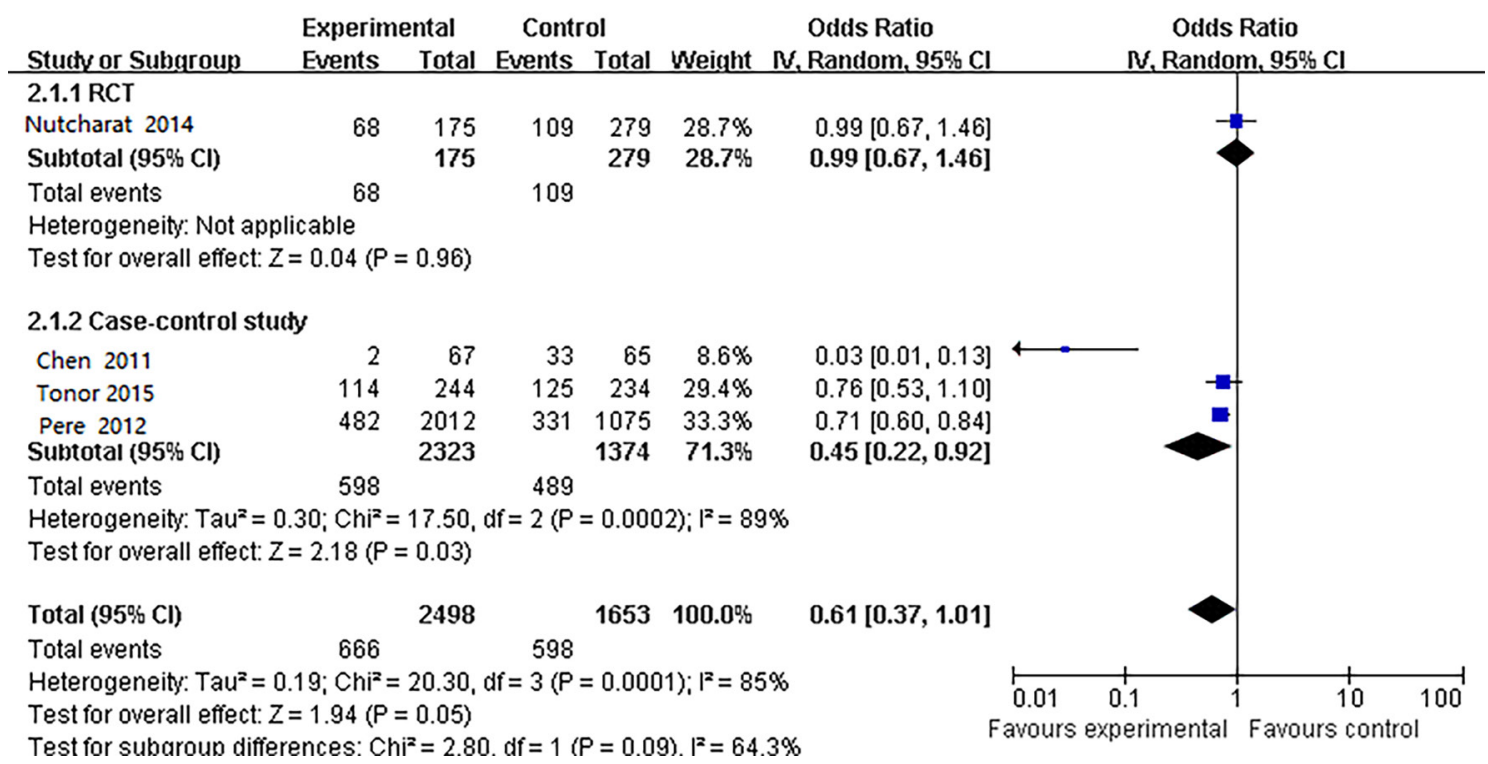

Figure 2 Comparison of the effect of hand washing over less than 4 times day $v s$. more than 4 times a day on disease prevention.

4 times per day may be a protective factor in preventing respiratory disease $(\mathrm{OR}=0.45,95 \% \mathrm{CI}: 0.22$ to 0.92$)$. The result of an RCT study (27) showed no statistical significance in the prevention effect $(\mathrm{OR}=0.99,95 \% \mathrm{CI}$ : 0.67 to 1.46 ) (Figure 2). Egger's test showed no publication bias $(\mathrm{P}=0.445)$, but the quality of evidence was graded low (Table S4).

\section{Pairwise comparison of hand washing $\leq 4$ times per day, 5-10 times per day, and $>10$ times per day}

A case-control study showed (14) that compared to hand washing $\leq 4$ times/day, washing hands $5-10$ times per day and $>10$ times per day could reduce the risk at the most influenza A infection ( $\mathrm{OR}=0.75,95 \% \mathrm{CI}$ : 0.63 to 0.91 ; OR $=0.65,95 \%$ CI: 0.53 to 0.80 , respectively). Hand washing 10 times a day may be better at preventing infection, there was no statistical significance between hand washing 10 times per day and 5-10 times per day (OR $=0.86,95 \%$ CI: 0.70 to 1.06 ) (Figure 3). The quality of evidence was graded low (Table $\mathrm{S} 4)$.

\section{$>10$ times per day versus $\leq 10$ times per day}

Two case-control studies $(14,15)$ compared the effect of hand washing more than 10 times per day to less than 10 times per day on respiratory disease prevention. The heterogeneity test showed that $\mathrm{I}^{2}=86 \%$, so the random- effects model was used. The results of the meta-analysis showed that compared with hand washing $\leq 10$ times/day, hand washing $>10$ times/day was a protective factor against respiratory disease infection $(\mathrm{OR}=0.59,95 \%$ CI: 0.36 to 0.97) (Figure 4). The quality of evidence was graded low (Table S4).

\section{7 times per day versus 4 times per day}

A randomized controlled trial study (20) showed that patients assigned to the intensive hand washing intervention group washed hands more frequently compared with the control group (7 versus 4 times a day, respectively) and developed fewer episodes of diarrheal illness (WMD $=-1.68,95 \%$ CI: -1.93 to -1.43 ) (Figure S1). The quality of evidence was graded low (Table S4).

\section{Comparison of other hand washing frequency}

A cohort study (37) showed that there were no significant decreases in acute respiratory tract infection (ARI) rates and influenza-like illness among adults with increased frequency of daily hand washing after adjusting for age, vaccination status, gender, educational level, occupational status, household size, and child contact. Comparing washing hands 2, 3 or 4 times per day, washing hands 5-9 times per day was associated with an adjusted ARI rates $\mathrm{RR}=1.08$, $95 \%$ CI 0.87 to $1.33 ; 10-19$ times with adjusted $\mathrm{RR}=1.22$, 


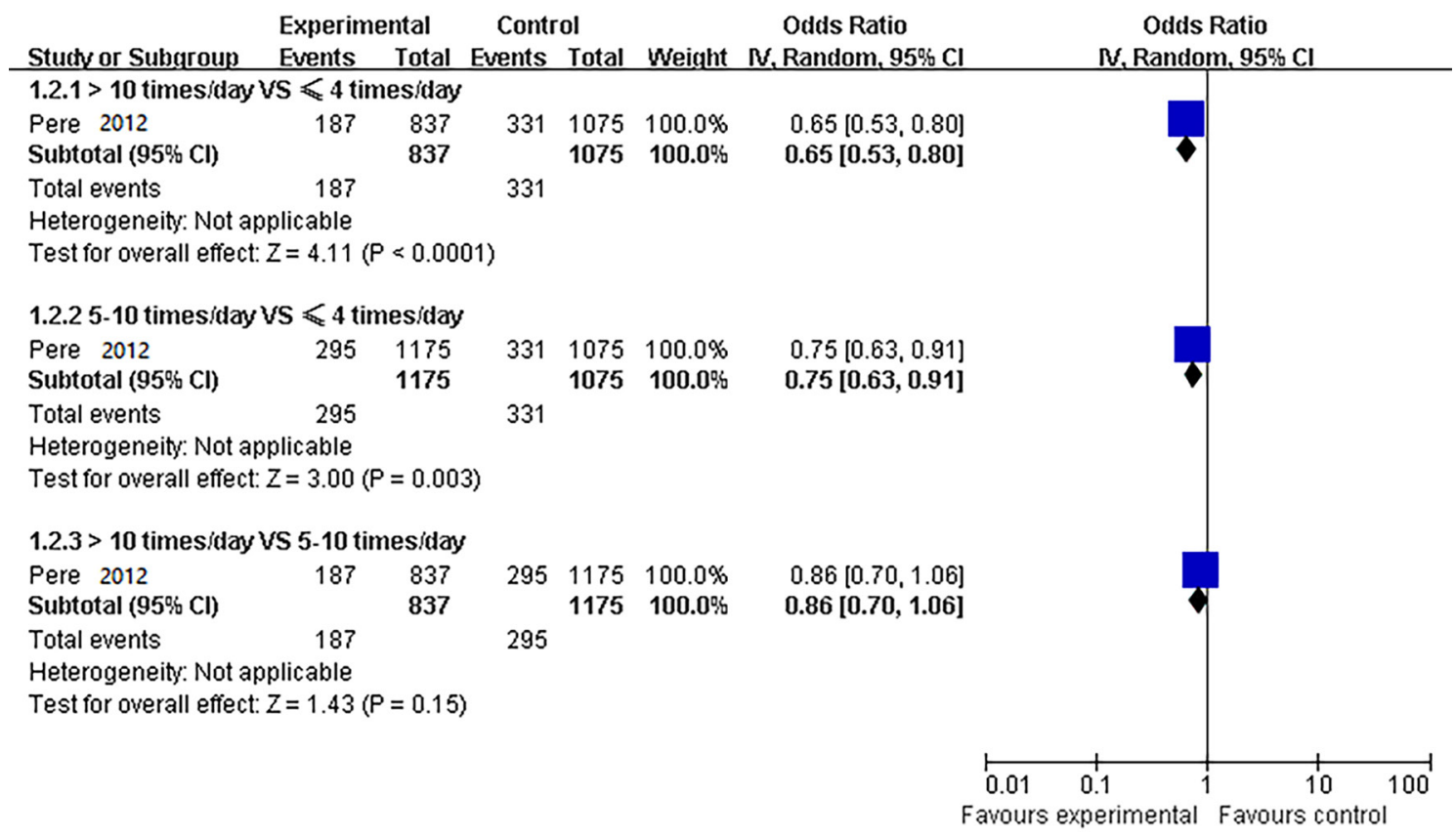

Figure 3 Comparison of different hand washing frequency for disease prevention.

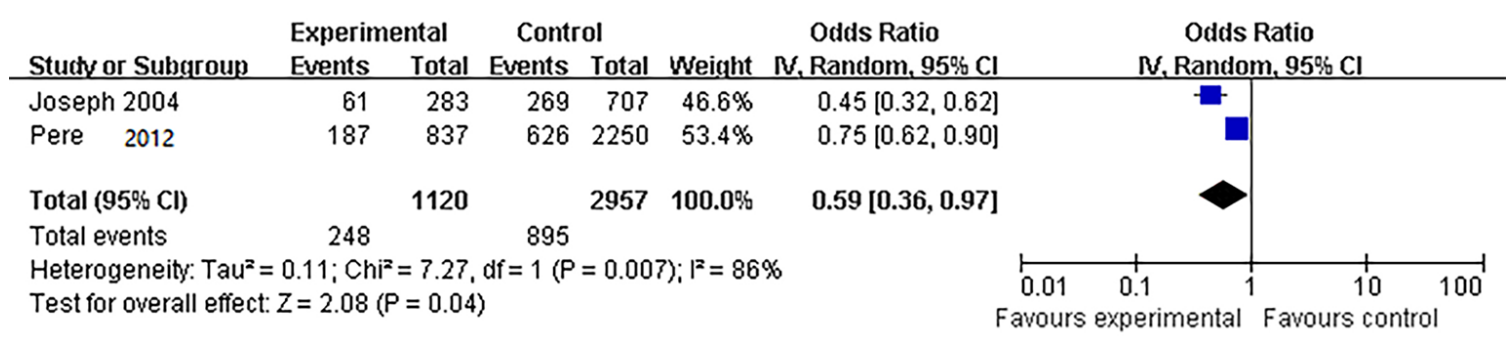

Figure 4 Comparison of the effect of hand washing more than 10 times per day on the prevention of respiratory diseases.

95\% CI: 0.97 to 1.53 ; and $\geq 20$ times with adjusted $R R=1.03$, 95\% CI: 0.81 to 1.32 . Washing hands 5-9 times per day was associated with an adjusted Influenza-like illness RR $=0.98$, 95\% CI: 0.66 to $1.46 ; 10-19$ times with adjusted $\mathrm{RR}=1.25$, $95 \%$ CI: 0.82 to 1.90 ; and $\geq 20$ times with adjusted RR $=1.06,95 \%$ CI: 0.68 to 1.67 . The quality of evidence was graded low (Table S4).

A cross-sectional study (38) showed no significant associations were found between hand washing frequency ( $\leq 3,3-6$ and $\geq 6$ times per day) and developing gastrointestinal or upper respiratory symptoms (runny nose adjusted $\mathrm{OR}=0.97,95 \% \mathrm{CI}$ : 0.64 to 1.49 ; itchy eyes adjusted $\mathrm{OR}=0.97,95 \% \mathrm{CI}: 0.53$ to 1.79 ; fever adjusted $\mathrm{OR}=0.61,95 \%$ CI: 0.25 to 1.47 ; Cough adjusted $\mathrm{OR}=0.98$,
95\% CI: 0.65 to 1.50 ; sore throat adjusted $\mathrm{OR}=1.42,95 \%$ CI: 0.93 to 2.16; Earache adjusted $\mathrm{OR}=1.42,95 \%$ CI: 0.66 to 3.02; Vomiting adjusted OR $=0.85,95 \%$ CI: 0.34 to 2.10 ; Diarrhea adjusted OR $=1.06,95 \%$ CI: 0.66 to 1.73 ). The quality of evidence was graded low (Table S4).

\section{Discussion}

A total of eight studies were included in this systematic review, when carefully analyzed showed generally no statistically significant difference in the impact of frequency of hand washing on disease occurrence or symptoms, although hand washing more than 4 times per day may be better than hand washing less than 4 times per day. 
A few low-quality pieces of evidence showed that hand washing more than 10 times per day could reduce the risk of respiratory and gastrointestinal diseases better than hand washing less than 10 times per day.

According to the results of this meta-analysis, hand washing more than 10 times per day can effectively reduce the risk of respiratory disease infection compared to hand washing less than 10 times per day, as determined in another study (39). Similar results have been observed in a previous systematic review, which pooled reviews from six case-control studies on varying public health interventions. The results showed that hand washing more than 10 times per day could effectively reduce the risk of the transmission of SARS (40). As new research has been published over the past decade, this issue needs to be reassessed. Additionally, as seen in the findings of this study, hand washing more than 10 times per day was better than more than 4 times per day in the prevention of the transmission of respiratory diseases, and the difference was found to be statistically significant. In other words, to some extent, it may be because insufficient hand washing frequency every day can't ensure the reducing the risk of diseases at critical moments. These critical moments include eating, inserting or removing contact lenses, preparing food, using the toilet, changing diapers or cleaning up children who have used the toilet, blowing your nose, coughing or snoring, contacting animals and their feed or waste, disposing of garbage, handling pet food, treating wounds or caring for patients and obviously dirty hands $(41,42)$.

Some studies have shown that hand hygiene is more effective in reducing gastrointestinal diseases than in preventing respiratory diseases $(43,44)$. Huang et al., for example, showed that hand washing 7 times per day versus 4 times per day resulted in fewer episodes of diarrheal illness (20). However, the study only included one gastrointestinal disease. Three studies showed no difference between different hand washing frequencies and the reducing the risk of respiratory diseases $(27,37,38)$. Two possible reasons may account for this result. On one hand, hand hygiene may have different effects on the prevention of different diseases. Multiple transmission routes of respiratory infections, for example, may mean that frequent hand washing may be ineffective. On the other hand, participants were students or adults in these three studies. However, in five other studies $(14,15,20,25,26)$, participants were patients and were matched to controls showed the opposite results. Therefore, hand washing frequency may have different effects on disease prevention for different groups.
The hand washing frequency may be affected by many factors, such as gender (38,45-47), age (45), economic conditions $(48,49)$, education level $(45,49)$, handwashing compliance $(50,51)$ and religious culture (52) etc. Being a female, middle-aged and having tertiary education level can effectively improve hand hygiene behavior (45). Females may be more likely to wash hands compared to males $(38,46,47)$. The data shows that after exposure to pollutants, handwashing frequency in high-income countries is higher $(48-72 \%)$, while that in low-income countries is only $5-25 \%$ (48). The low compliance of medical staff and the general public (22-59.6\%) can explain low handwashing frequency to some degree $(50,51)$.

The compliance of hand hygiene is influenced by the expected results of preventive measures, the perception of the ability of hand hygiene, and habit (52). A clear description of the effectiveness of protective actions helps the public actively prevent pandemics (53). A systematic review showed that setting long-term hand washing reminders or regular repeated feedback can improve the enthusiasm of medical staff to wash their hands (54). The implementation of health education to promote hand hygiene can effectively support children, medical staff, and patients to improve their hand washing frequency and technique (55-60). At the same time, improvements in hand washing facilities can also increase the frequency of hand washing, such as the availability of soap or hand sanitizer, as well as a clean napkin to dry hands $(41,42)$. Based on these differences, health workers need to develop a detailed education plan to improve people's enthusiasm for handwashing, so as to increase the handwashing frequency. These can refer to the WHO and the CDC in their hand hygiene guidelines and website materials and the need for the user (e.g., hospital) to adapt it to their culture and think of hand hygiene as a whole system $(41,42)$.

\section{Strengths and limitations}

This study is the first systematic review to specifically explore different frequencies of hand washing on the prevention of certain diseases, which provides evidence for recommendations on the most advantageous number of times to wash hands in a day and also provides directional guidance for future research. However, this study still has its limitations. Currently, there are few high-quality studies on hand washing frequency and disease prevention. Thus, the overall number of studies included in the analysis was small. Additionally, the impact of hand washing is influenced by 
many confounding factors (such as the standardization of hand washing methods, the cleaning agents used and their formulation, dose, delivery system, wash time, religious culture, and the risk of disease exposure in the population, etc.), which may reduce the reliability of the results.

It is recommended that future large-scale, high-quality hand washing frequency studies be conducted among different populations and different diseases. Besides, for the same population, the same disease prevention test should verify whether there is a certain frequency range relationship between the effect of handwashing frequency on disease prevention.

\section{Conclusions}

The results of this study indicate that the higher the frequency of hand washing, the better the effect on disease prevention. No high-quality evidence, however, indicates the best range of hand washing frequency for disease prevention. Hand hygiene is one of the basic components of infection control procedures, as washing hands frequently is a low-cost and high-efficacy health measure. Health promotion efforts should aim to expand hand hygiene education, increase public enthusiasm for washing hands, and improve health.

\section{Acknowledgments}

We thank Sarah Robbins (Institute of Bill \& Melinda Gates Foundation), Shu Yang (Chengdu University of Traditional Chinese Medicine) for providing guidance and comments for our review. We thank all the authors for their wonderful collaboration.

Funding: This work was supported by grants from the Fundamental Research Funds for the Central Universities (lzujbky-2020-sp14); Special funding for prevention and control of emergency of COVID-19 from Key Laboratory of Evidence Based Medicine and Knowledge Translation of Gansu Province (grant number No. GSEBMKT-2020YJ01) to YC.

\section{Footnote}

Reporting Checklist: The authors have completed the PRISMA reporting checklist. Available at http://dx.doi. org/10.21037/atm-20-6005

Peer Review File: Available at http://dx.doi.org/10.21037/ atm-20-6005
Conflicts of Interest: All authors have completed the ICMJE uniform disclosure form (available at http://dx.doi. org/10.21037/atm-20-6005). The authors have no conflicts of interest to declare.

Ethical Statement: The authors are accountable for all aspects of the work in ensuring that questions related to the accuracy or integrity of any part of the work are appropriately investigated and resolved.

Open Access Statement: This is an Open Access article distributed in accordance with the Creative Commons Attribution-NonCommercial-NoDerivs 4.0 International License (CC BY-NC-ND 4.0), which permits the noncommercial replication and distribution of the article with the strict proviso that no changes or edits are made and the original work is properly cited (including links to both the formal publication through the relevant DOI and the license). See: https://creativecommons.org/licenses/by-nc-nd/4.0/.

\section{References}

1. Ejemot-Nwadiaro RI, Ehiri JE, Arikpo D, et al. Hand washing promotion for preventing diarrhoea. Cochrane Database Syst Rev 2015;2015:CD004265.

2. Yen MY, Lin YE, Su IJ, et al. Using an integrated infection control strategy during outbreak control to minimize nosocomial infection of severe acute respiratory syndrome among healthcare workers. J Hosp Infect 2006;62:195-9.

3. Jefferson T, Del Mar C B, Dooley L, et al. Physical interventions to interrupt or reduce the spread of respiratory viruses. Cochrane Database Syst Rev 2011;2011:CD006207.

4. Cowling B J, Chan K H, Fang V J, et al. Facemasks and Hand Hygiene to Prevent Influenza Transmission in Households. Ann Intern Med 2009; 151:437.

5. Chen S, Yang J, Yang W, et al. COVID-19 control in China during mass population movements at New Year. Lancet 2020;395:764-6.

6. GBD 2016 Diarrhoeal Disease Collaborators. Estimates of the global, regional, and national morbidity, mortality, and aetiologies of diarrhoea in 195 countries: a systematic analysis for the Global Burden of Disease Study 2016. Lancet Infect Dis 2018;18:1211-28.

7. Liu L, Oza S, Hogan D, et al. Global, regional, and national causes of child mortality in 2000-13, with projections to inform post-2015 priorities: an updated systematic analysis. Lancet 2015;385:430-40. 


\section{Page 10 of 11}

8. Li Q, Guan X, Wu P, et al. Early Transmission Dynamics in Wuhan, China, of Novel Coronavirus-Infected Pneumonia. N Engl J Med 2020;382:1199-207.

9. Liu L, Johnson HL, Cousens S, et al. Global, regional, and national causes of child mortality: an updated systematic analysis for 2010 with time trends since 2000. Lancet 2012;379:2151-61.

10. Vestergaard LS, Nielsen J, Krause TG, et al. Excess allcause and influenza-attributable mortality in Europe, December 2016 to February 2017. Euro surveill 2017;22:30506.

11. Allegranzi B, Pittet D. Role of hand hygiene in healthcare-associated infection prevention. J Hosp Infect 2009; 73:305-15.

12. Centers for Disease Control and Prevention (2017). Clean hands save lives. Available online: https://www.cdc.gov/ handwashing/index.html

13. Bloomfield SF, Aiello AE, Cookson B, et al. The effectiveness of hand hygiene procedures in reducing the risks of infections in home and community settings including handwashing and alcohol-based hand sanitizers. Am J Infect Control 2007;35:S27-S64.

14. Godoy P, Castilla J, Delgado-Rodríguez M, et al. Effectiveness of hand hygiene and provision of information in preventing influenza cases requiring hospitalization. Prev Med 2012;54:434-9.

15. Lau JTF, Tsui H, Lau M, et al. SARS transmission, risk factors, and prevention in Hong Kong. Emerg Infect Dis 2004;10:587-92.

16. Ejemot-Nwadiaro RI, Ehiri JE, Meremikwu MM, et al. Hand washing for preventing diarrhoea. Cochrane Database Syst Rev 2015;2015:CD004265.

17. Aiello AE, Coulborn RM, Perez V, et al. Effect of Hand Hygiene on Infectious Disease Risk in the Community Setting: A Meta-Analysis. Am J Public Health 2008;98:1372-81.

18. Freeman MC, Stocks ME, Cumming O, et al. Hygiene and health: systematic review of handwashing practices worldwide and update of health effects. Trop Med Int Health 2014;19:906-16.

19. Ejemot RI, Ehiri JE, Meremikwu MM, et al. Cochrane review: Hand washing for preventing diarrhoea. Evid Based Child Health 2009;4:893-939.

20. Huang DB, Zhou J. Effect of intensive handwashing in the prevention of diarrhoeal illness among patients with AIDS: a randomized controlled study. J Med Microbiol 2007;56:659-63.

21. Rabie T, Curtis V. Handwashing and risk of respiratory

\section{Xun et al. Hand washing frequency and the risk of disease}

infections: a quantitative systematic review. Trop Med Int Health 2006;11:258-67.

22. Wang Z, Lapinski M, Quilliam E, et al. The effect of hand-hygiene interventions on infectious disease-associated absenteeism in elementary schools: A systematic literature review. Am J Infect Control 2017;45:682-9.

23. World Health Organization. Coronavirus disease (COVID-19) advice for the public. 2020. Available online: https://www.who.int/zh/emergencies/diseases/novelcoronavirus-2019/advice-for-public

24. Alba S, Bakker MI, Hatta M, et al. Risk Factors of Typhoid Infection in the Indonesian Archipelago. PLoS One 2016;11:e0155286.

25. Chen JD, Yuan J, He Z, et al. A case-control study of an influenza A (H1N1) outbreak in a hospital. Zhonghua Yu Fang Yi Xue Za Zhi 2011;45:101-6.

26. Torner N, Soldevila N, Garcia JJ, et al. Effectiveness of nonpharmaceutical measures in preventing pediatric influenza: a case-control study. BMC Public Health 2015;15:543.

27. Mangklakeree N, Pinitsoontorn S, Srisaenpang S. Brief communication Effectiveness of influenza control using nonpharmaceutical interventions at primary schools in Nakhon Phanom Province, Northeast Thailand. Asian Biomed 2014;3:405-10.

28. Higgins JPT, Altman DG, Gøtzsche PC, et al. The Cochrane Collaboration's tool for assessing risk of bias in randomised trials. BMJ 2011;343:d5928.

29. Wells G, Shea B, O'Connell J. The Newcastle-Ottawa Scale (NOS) for Assessing The Quality of Nonrandomised Studies in Meta-analyses. Ottawa Health Research Institute Web site 2014;7.

30. Rostom A, Dubé C, Cranney A, et al. Celiac disease. Evid Rep Technol Assess (Summ) 2004;(104):1-6.

31. Review Manager (RevMan) [Computer program]. Version 5.3. Copenhagen: The Nordic Cochrane Centre, The Cochrane Collaboration, 2014.

32. GRADEpro G D T. GRADEpro Guideline Development Tool [Software]. McMaster University, 2015 (developed by Evidence Prime, Inc.). Available online: www.gradepro.org

33. Guyatt GH, Oxman AD, Montori V, et al. GRADE guidelines: 5 . Rating the quality of evidence-publication bias. J Clin Epidemiol 2011;64:1277-82.

34. Guyatt GH, Oxman AD, Kunz R, et al. GRADE guidelines 6. Rating the quality of evidence-imprecision. J Clin Epidemiol 2011;64:1283-93.

35. Guyatt GH, Oxman AD, Kunz R, et al. GRADE guidelines: 7. Rating the quality of evidenceinconsistency. J Clin Epidemiol 2011;64:1294-302. 
36. Guyatt GH, Oxman AD, Kunz R, et al. GRADE guidelines: 8. Rating the quality of evidence-indirectness. J Clin Epidemiol 2011;64:1303-10.

37. Merk H, Kuhlmann-Berenzon S, Linde A, et al. Associations of hand-washing frequency with incidence of acute respiratory tract infection and influenza-like illness in adults: a population-based study in Sweden. BMC Infect Dis 2014;14:509.

38. Thumma J, Aiello AE, Foxman B. The association between handwashing practices and illness symptoms among college students living in a university dormitory. Am J Infect Control 2009;37:70-2.

39. Lau JTF, Griffiths S, Choi K-c, et al. Prevalence of preventive behaviors and associated factors during early phase of the H1N1 influenza epidemic. Am J Infect Control 2010;38:374-80.

40. Jefferson T, Del Mar C, Dooley L, et al. Physical interventions to interrupt or reduce the spread of respiratory viruses: systematic review. BMJ 2009;339:b3675.

41. World Health Organization. WHO guidelines on hand hygiene in health care. Available online: https://www.cdc. gov/handwashing/when-how-handwashing.html

42. Mayoclinic. Healthy Lifestyle. Available online: https:// www.mayoclinic.org/healthy-lifestyle/adult-health/indepth/hand-washing/art-20046253

43. Aiello AE, Larson EL. What is the evidence for a causal link between hygiene and infections? Lancet Infect Dis 2002;2:103-10.

44. Aiello AE, Larson EL, Levy SB. Consumer Antibacterial Soaps: Effective or Just Risky? Clin Infect Dis 2007;45:S137-47.

45. Suen LKP, So ZYY, Yeung SKW, et al. Epidemiological investigation on hand hygiene knowledge and behavior: a cross-sectional study on gender disparity. BMC Public Health 2019;19:401.

46. van de Mortel T, Bourke R, McLoughlin J, et al. Gender influences handwashing rates in the critical care unit. Am J Infect Control 2001;29:395-9.

47. Edwards D, Monk-Turner E, Poorman S, et al. Predictors of hand-washing behavior. Soc Behav Pers 2002;30:751-6.

Cite this article as: Xun Y, Shi Q, Yang N, Yang N, Li Y, Si W, Shi Q, Wang Z, Liu X, Yu X, Zhou Q, Yang M, Chen Y. Associations of hand washing frequency with the incidence of illness: a systematic review and meta-analysis. Ann Transl Med 2021;9(5):395. doi: 10.21037/atm-20-6005
48. Freeman MC, Stocks ME, Cumming O, et al. Systematic review: Hygiene and health: systematic review of handwashing practices worldwide and update of health effects. Trop Med Int Health 2014;19:906-16.

49. Tao SY, Cheng YL, Lu Y, et al. Handwashing behavior among Chinese adults: a cross-sectional study in five provinces. Public Health 2013;127:620-8.

50. Clark J, Henk JK, Crandall PG, et al. An observational study of handwashing compliance in a child care facility. Am J Infect Control 2016;44:1469-74.

51. Lambe KA, Lydon S, Madden C, et al. Hand Hygiene Compliance in the ICU: A Systematic Review. Crit Care Med 2019;47:1251-7.

52. Erasmus V, Otto S, De Roos E, et al. Assessment of correlates of hand hygiene compliance among final year medical students: a cross-sectional study in the Netherlands. BMJ Open 2020;10:e029484.

53. Teasdale E, Yardley L, Schlotz W, et al. The importance of coping appraisal in behavioural responses to pandemic flu. Br J Health Psychol 2012;17:44-59.

54. Naikoba S, Hayward A. The effectiveness of interventions aimed at increasing handwashing in healthcare workers - a systematic review. J Hosp Infect 2001;47:173-80.

55. Wang J, Kuang C, Gu W, et al. Evaluation and analysis of the effect of hand hygiene intervention for infants in Shenzhen. J Med Pes Control 2020;36:135-7.

56. Witta SD, Spencer HA. Using educational interventions to improve the handwashing habits of preschool children. Early Child Dev Care 2004;174:461-71.

57. Lee B, Newberg A. Religion and health: a review and critical analysis. Zygon. 2005;40:443-68.

58. Ding C. Effect of comprehensive intervention measures on hand hygiene compliance of nurses in infectious wards. J Clin Med 2018;5:127-8.

59. Kong X, Wang Z, Zhu X. Research on the Effect of Intuitive Education Method on Improving Medical Workers' Hand Hygiene Compliance. Contemp Clin Med 2016;29:2323-4.

60. Zhang Y, Du Y. Intervention strategy of hand hygiene compliance in intestinal clinic patients. Chin J Mod Med 2019;21:100-2. 
Appendix 1 Search strategy

PubMed (N=1294) (bttps://pubmed.ncbi.nlm.nib.gov)

\#1. $\quad$ "Preventive Health Services"[Mesh]

\#2. "Preventive Medicine"[Mesh]

\#3. $\quad$ "prevention and control"[Subheading]

\#4. "preventive therapy"[Title/Abstract]

\#5. "preventive measures"[Title/Abstract])

\#6. Prevent ${ }^{\star}[$ Title/Abstract]

\#7. safeguard[Title/Abstract]

\#8. precaution[Title/Abstract]

\#9. defense[Title/Abstract]

\#10. prophylaxis[Title/Abstract]

\#11. "prevention and control"[Title/Abstract]

\#12. \#1-\#11/OR

\#13. "Handwashing"[Mesh]

\#14. "hand washing*" [Title/Abstract]

\#15. "hand-washing" [Title/Abstract]

\#16. "hand-cleaning" [Title/Abstract]

\#17. "hand hygiene" [Title/Abstract]

\#18. "wash* hand" [Title/Abstract]

\#19. "clean hand*" [Title/Abstract]

\#20. \#13-\#19/OR

\#21. Frequency [All fields]

\#22. Times[All fields]

\#23. Seldom[All fields]

\#24. Always[All fields]

$\# 25$. often[All fields]

\#26. \#21-\#25/OR

\#27. \#12 AND \#20 AND \#26

Cochrane library (N=133) (bttps://www.cochranelibrary. com/)

\#1. MeSH descriptor: [Preventive Health Services] explode all trees

\#2. MeSH descriptor: [Preventive Medicine] explode

all trees

\#3. Prevent*:ti,ab,kw

\#4. safeguard:ti,ab,kw

\#5. precaution:ti,ab,kw

\#6. defense:ti,ab,kw

\#7. prophylaxis:ti,ab,kw

\#8. $\quad$ "prevention and control":ti,ab,kw

\#9. "preventive therapy":ti,ab,kw

\#10. "preventive measures":ti,ab,kw

\#11. \#1-\#10/OR

\#12. MeSH descriptor: [hand hygiene] explode all trees
\#13. "hand washing*":ti,ab,kw

\#14. "hand-washing":ti,ab,kw

\#15. "hand-cleaning":ti,ab,kw

\#16. "wash* hand":ti,ab,kw

\#17. "clean hand":ti,ab,kw

\#18. \#12-\#17/OR

\#19. Frequency

\#20. Times

\#21. seldom

\#22. always

\#23. often

\#24. \#19-\#23/OR

\#25. \#11 AND \#18 AND \#24

Web of Science (N=962) (bttps://apps.webofknowledge. com/)

\#1. TOPIC: "Prevent ${ }^{* "}$

\#2. TOPIC: "safeguard"

\#3. TOPIC: "precaution"

\#4. TOPIC: "defense"

\#5. TOPIC: "prophylaxis"

\#6. TOPIC: "prevention and control"

\#7. TOPIC: "preventive therapy"

\#8. TOPIC: "preventive measures"

\#9. \#1-\#8/OR

\#10. TOPIC: "Handwashing*"

\#11. TOPIC: "hand washing*"

\#12. TOPIC: "hand-washing"

\#13. TOPIC: "hand-cleaning"

\#14. TOPIC: "hand hygiene"

\#15. TOPIC: "wash* hand*"

\#16. TOPIC: "clean hand*"

\#17. \#10-\#16/OR

\#18. All fields: "Frequency"

\#19. All fields: "times"

\#20. All fields: "seldom"

\#21. All fields: "always"

\#22. All fields: "often"

\#23. \#18-\#22/OR

\#24. \#9 AND \#17 AND \#23

Embase (N=1349) (bttps://www.embase.com/)

\#1. 'prevention and control'/exp

\#2. 'defense'/exp

\#3. 'prophylaxis'/exp

\#4. 'Prevent*':ab,ti 

\#5. 'safeguard ':ab,ti
\#6. 'precaution ':ab,ti
\#7. ' preventive therapy':ab,ti
\#8. 'preventive measures':ab,ti
\#9. \#1-\#8/OR
\#10. 'hand washing'/exp
\#11. 'handwashing ${ }^{* '}: a b, t i$
\#12. 'hand washing ${ }^{* \prime}: a b, t i$
\#13. 'hand-washing':ab,ti
\#14. 'hand-cleaning':ab,ti
\#15. 'hand hygiene':ab,ti
\#16. 'wash* hand':ab,ti
\#17. 'clean hand"':ab,ti
\#18. \#10-\#17/OR
\#19. frequency
\#20. times
\#21. seldom
\#22. always
\#23. often
\#24. \#19-\#23/OR
\#25. \#9 AND \#18 AND \#24

\section{CBM (N=1376) (bttp://www.sinomed.ac.cn)}

\#1. “预防” [ 常用字段 : 智能 ]

\#2. “防控”[ 常用字段 : 智能]

\#3. “防护” [ 常用字段 : 智能 ]

\#4. “防治”[ 常用字段 : 智能 ]

\#5. “防患”[ 常用字段 : 智能 ]

\#6. “防御” [ 常用字段 : 智能 ]

\#7. “防卫”[ 常用字段 : 智能 ]

\#8. “防范”[ 常用字段 : 智能 ]

\#9. “公共卫生”[ 不加权 : 扩展 ]

\#10. “预防卫生服务”[ 不加权 : 扩展 ]

\#11. \#1-\#10/0R

\#12. “手卫生” [ 不加权 : 扩展 ]

\#13. “洗手” " 常用字段 : 智能 ]

\#14. “手部清洁 " [ 常用字段 : 智能 ]

\#15. \#12-\#14/0R

\#16. “次” [ 全部字段 : 智能 ]

\#17. “频率”[ 全部字段 : 智能 ]

\#18. \#16 OR \#17
\#19. \#11 AND \#15 AND \#18

Wan Fang (N=1421) (bttp://www.wanfangdata.com.cn)

\#1. “预防”[ 主题 ]

\#2. “防控”[主题 ]

\#3. “防护”[ 主题 ]

\#4. “防治”[ 主题 ]

\#5. “防患”[主题 ]

\#6. “防御”[主题 ]

\#7. “防卫”[ 主题 ]

\#8. “防范”[ 主题 ]

\#9. \#1-\#8/0R

\#10. “洗手”[ 主题 ]

\#11. “手卫生”[主题 ]

\#12. “手部清洁”[ 主题 ]

\#13. \#10-\#12/0R

\#14. “次”[ 全部 ]

\#15. " 频率” [ 全部 ]

\#16. \#14 OR \#15

\#17. \#9 AND \#13 AND \#16

\section{CNKI (N=2029) (bttps://www.cnki.net)}

\#1. 预防 [ 主题 ]

\#2. 防控 [ 主题 ]

\#3. 防护 [ 主题 ]

\#4. 防治 [ 主题 ]

\#5. 防患 [ 主题 ]

\#6. 防御 [主题 ]

\#7. 防卫 [主题 ]

\#8. 防范 [ 主题 ]

\#9. \#1-\#8/0R

\#10. 洗手 [ 主题 ]

\#11. 手卫生 [主题 ]

\#12. 手部清洁 [ 主题 ]

\#13. \#10-\#12/0R

\#14. 次 [ 全文]

\#15. 频率 [ 全文 ]

\#16. \#14 OR \#15

\#17. \#9 AND \#13 AND \#16 


\begin{tabular}{|c|c|c|c|c|c|c|}
\hline Study & Random sequence generation & Allocation concealment & $\begin{array}{l}\text { Blinding of participants and personnel; } \\
\text { Blinding of outcome assessment }\end{array}$ & $\begin{array}{l}\text { Incomplete outcome data. Assessments should be } \\
\text { made for each main outcome (or class of outcomes) }\end{array}$ & Selective reporting & Other sources of bias \\
\hline Huang 2007, (20) & Low & Unclear & Unclear & Low & Unclear & Unclear \\
\hline Mangklakeree 2014, (27) & Low & Unclear & Unclear & Low & Unclear & Low \\
\hline
\end{tabular}

Table S2 The risk of bias of cross-sectional studies

Bias domains include in Agency for Healthcare Research and Quality (AHRQ)

\begin{tabular}{|c|c|c|c|c|c|c|c|c|c|c|c|}
\hline \multirow[b]{2}{*}{ Study } & \multicolumn{11}{|c|}{ Bias domains include in Agency for Healthcare Research and Quality (AHRQ) } \\
\hline & $\begin{array}{l}\text { Define the source } \\
\text { of information } \\
\text { (survey, record } \\
\text { review) }\end{array}$ & $\begin{array}{l}\text { List inclusion and } \\
\text { exclusion criteria } \\
\text { for exposed } \\
\text { and unexposed } \\
\text { subjects (case } \\
\text { and controls) or } \\
\text { refer to previous } \\
\text { publications }\end{array}$ & $\begin{array}{l}\text { Indicate time period } \\
\text { used for identifying } \\
\text { patients }\end{array}$ & $\begin{array}{l}\text { Indicate whether or } \\
\text { not subjects were } \\
\text { consecutive if not } \\
\text { population-based }\end{array}$ & $\begin{array}{l}\text { Indicate if evaluators } \\
\text { of subjective } \\
\text { components of study } \\
\text { were masked to other } \\
\text { aspects of the status } \\
\text { of the participants }\end{array}$ & $\begin{array}{l}\text { Describe any } \\
\text { assessments } \\
\text { undertaken for } \\
\text { quality assurance } \\
\text { purposes (e.g., } \\
\text { test/retest of } \\
\text { primary outcome } \\
\text { measurements) }\end{array}$ & $\begin{array}{l}\text { Explain any patient } \\
\text { exclusions from } \\
\text { analysis }\end{array}$ & $\begin{array}{l}\text { Describe how } \\
\text { confounding was } \\
\text { assessed and/or } \\
\text { controlled. }\end{array}$ & $\begin{array}{l}\text { If applicable, } \\
\text { explain how } \\
\text { missing data } \\
\text { were handled in } \\
\text { the analysis }\end{array}$ & $\begin{array}{l}\text { Summarize patient } \\
\text { response rates and } \\
\text { completeness data } \\
\text { collection }\end{array}$ & $\begin{array}{l}\text { Clarify what follow-up, } \\
\text { if any, was expected } \\
\text { and the percentage } \\
\text { of patients for which } \\
\text { in complete data or } \\
\text { follow-up was obtained }\end{array}$ \\
\hline $\begin{array}{l}\text { Thumma } \\
\text { 2009, (38) }\end{array}$ & Yes & Yes & No & Yes & Unclear & Unclear & Yes & Yes & Yes & No & No \\
\hline
\end{tabular}

Table S3 The risk of bias of cohort and case-control studies

\begin{tabular}{|c|c|c|c|c|c|c|c|c|c|c|}
\hline \multirow[b]{3}{*}{ Study } & \multicolumn{10}{|c|}{ Bias domains include in Newcastle-Ottawa Quality Assessment Scale } \\
\hline & \multicolumn{4}{|c|}{ Selection } & \multicolumn{2}{|r|}{ Comparability } & \multicolumn{3}{|c|}{ Exposure } & \multirow[b]{2}{*}{ Total points } \\
\hline & $\begin{array}{l}\text { Is the case definition } \\
\text { adequate? }\end{array}$ & $\begin{array}{l}\text { Representativeness of } \\
\text { the cases }\end{array}$ & Selection of controls & Definition of controls & $\begin{array}{l}\text { Study controls } \\
\text { for--select the most } \\
\text { important factor }\end{array}$ & $\begin{array}{l}\text { Study controls for any additional } \\
\text { factor (the criteria could be modified } \\
\text { to indicate specific control for a } \\
\text { second important factor }\end{array}$ & $\begin{array}{l}\text { Ascertainment } \\
\text { of expose }\end{array}$ & $\begin{array}{l}\text { Same method of } \\
\text { ascertainment for } \\
\text { case and controls }\end{array}$ & Non-Response rate & \\
\hline $\begin{array}{l}\text { Godoy } \\
\text { 2012, (14) }\end{array}$ & $\star$ & $\star$ & $\star$ & 太 & 太 & « & 太 & $\star$ & .. & $8 \star$ \\
\hline $\begin{array}{l}\text { Lau } \\
\text { 2004, (15) }\end{array}$ & $\star$ & $\star$ & $\star$ & $\star$ & $\star$ & & $\star$ & $\star$ & .. & $7 \star$ \\
\hline $\begin{array}{l}\text { Torner } \\
\text { 2015, (26) }\end{array}$ & $\star$ & $\star$ & $\star$ & $\star$ & $\star$ & $\star$ & .. & $\star$ & $\star$ & $8 \star$ \\
\hline $\begin{array}{l}\text { Chen } \\
\text { 2011, (25) }\end{array}$ & $\star$ & $\star$ & .. & $\star$ & $\star$ & $\star$ &.. & $\star$ & $\star$ & $7 \star$ \\
\hline $\begin{array}{l}\text { Merk } \\
\text { 2014, (37) }\end{array}$ & $\star$ & .. & $\star$ & .. & $\star$ & $\star$ & $\star$ & $\star$ & $\star$ & $7 \star$ \\
\hline
\end{tabular}

Newcastle-Ottawa Scale is a 'star system' and a star $(\star)$ means that the content of the study matches this item. 
Table S4 GRADE evidence profile

\begin{tabular}{|c|c|c|c|c|c|c|c|c|c|c|}
\hline \multirow{2}{*}{ No. of studies } & \multicolumn{5}{|c|}{ Certainty assessment } & \multicolumn{3}{|c|}{ No. of patients } & \multirow{2}{*}{$\begin{array}{l}\text { Effect value } \\
(95 \% \mathrm{Cl})\end{array}$} & \multirow{2}{*}{ Certainty } \\
\hline & Risk of bias & Inconsistency & Indirectness & Imprecision & Other considerations & Sample & Intervention & Control & & \\
\hline \multicolumn{11}{|c|}{ Prevent infections from diseases } \\
\hline \multicolumn{11}{|c|}{$\leq 4$ times/day vs. $>4$ times/day } \\
\hline $\mathrm{RCT}(1)$ & Serious ${ }^{1}$ & Serious $^{2}$ & Not serious & Not serious & None & 454 & 175 & 279 & $\begin{array}{l}\text { OR } 0.99 ; \\
\text { (0.67 to } 1.46)\end{array}$ & $\begin{array}{c}\oplus \oplus \bigcirc \bigcirc \\
\text { LOW }\end{array}$ \\
\hline $\operatorname{ccs}(3)$ & Not serious & Serious $^{2}$ & Not serious & Not serious & None & 3,697 & 1,087 & 2,610 & $\begin{array}{c}\text { OR } 0.45 ; \\
(0.22 \text { to } 0.92)\end{array}$ & $\begin{array}{c}\oplus \oplus \bigcirc \bigcirc \\
\text { LOW }\end{array}$ \\
\hline \multicolumn{11}{|c|}{$\leq 4$ times/day vs. $5-10$ times/day } \\
\hline $\operatorname{ccs}(1)$ & Serious $^{1}$ & Not serious & Not serious & Not serious & None & 2,250 & 626 & 1,624 & $\begin{array}{l}\text { OR } 0.75 ; \\
\text { (0.63 to } 0.91)\end{array}$ & $\begin{array}{c}\oplus \oplus \bigcirc \bigcirc \\
\text { LOW }\end{array}$ \\
\hline \multicolumn{11}{|c|}{$\leq 4$ times/day vs. $>10$ times/day } \\
\hline $\operatorname{ccs}(1)$ & Serious $^{1}$ & Not serious & Not serious & Not serious & None & 1,912 & 518 & 1,394 & $\begin{array}{l}\text { OR } 0.65 ; \\
(0.53 \text { to } 0.80)\end{array}$ & $\begin{array}{c}\oplus \oplus \bigcirc \bigcirc \\
\text { LOW }\end{array}$ \\
\hline \multicolumn{11}{|c|}{$5-10$ times/day vs. $>10$ times/day } \\
\hline $\operatorname{ccs}(1)$ & Serious $^{1}$ & Not serious & Not serious & Not serious & None & 2,012 & 482 & 15,30 & $\begin{array}{c}\text { OR } 0.86 ; \\
(0.70 \text { to } 1.06)\end{array}$ & $\begin{array}{c}\oplus \oplus \bigcirc \bigcirc \\
\text { LOW }\end{array}$ \\
\hline \multicolumn{11}{|c|}{$\leq 10$ times/day vs. $>10$ times/day } \\
\hline $\operatorname{ccs}(2)$ & Serious $^{1}$ & Serious $^{2}$ & Not serious & Not serious & None & 4,077 & 1,143 & 2,934 & $\begin{array}{l}\text { OR } 0.59 \text {; } \\
\text { (0.36 to } 0.97)\end{array}$ & $\begin{array}{c}\oplus \oplus \bigcirc \bigcirc \\
\text { LOW }\end{array}$ \\
\hline \multicolumn{11}{|c|}{ 2-4 times/day vs. 5-9 times/day } \\
\hline $\operatorname{CS}(1)$ & Not serious & Not serious & Not serious & Not serious & None & 1,532 & 351 & 1,181 & $\begin{array}{c}\text { RR 1.08; } \\
\text { (0.87 to } 1.33)\end{array}$ & $\begin{array}{c}\oplus \oplus \bigcirc \bigcirc \\
\text { LOW }\end{array}$ \\
\hline \multicolumn{11}{|c|}{ 2-4 times/day vs. $10-19$ times/day } \\
\hline $\operatorname{CS}(1)$ & Not serious & Not serious & Not serious & Not serious & None & 1,119 & 351 & 768 & $\begin{array}{l}\text { RR 1.22; } \\
\text { (0.97 to } 1.53)\end{array}$ & $\begin{array}{c}\oplus \oplus \bigcirc \bigcirc \\
\text { LOW }\end{array}$ \\
\hline \multicolumn{11}{|c|}{$2-4$ times/day vs. $\geq 20$ times } \\
\hline CS (1) & Not serious & Not serious & Not serious & Not serious & None & 915 & 351 & 564 & $\begin{array}{l}\text { RR 1.03; } \\
\text { (0.81 to } 1.32 \text { ) }\end{array}$ & $\begin{array}{c}\oplus \oplus \bigcirc \bigcirc \\
\text { LOW }\end{array}$ \\
\hline \multicolumn{11}{|c|}{ Episodes of diarrheal illness } \\
\hline \multicolumn{11}{|c|}{4 times/day vs. 7 times/day } \\
\hline $\mathrm{RCT}(1)$ & Serious $^{1}$ & Not serious & Serious $^{3}$ & Not serious & None & 148 & 75 & 73 & $\begin{array}{l}\text { WMD -1.68; } \\
\text { (-1.93 to }-1.43)\end{array}$ & $\begin{array}{c}\oplus \oplus \bigcirc \bigcirc \\
\text { LOW }\end{array}$ \\
\hline
\end{tabular}

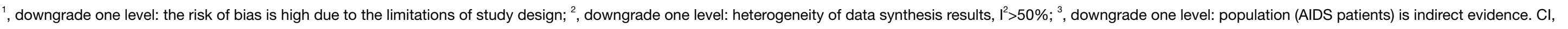
confidence interval; OR, odd ratio; RR, risk ratio; WMD, weighted mean difference; RCT, randomized controlled trial; CCS, case-control study; CS, cohort study. 


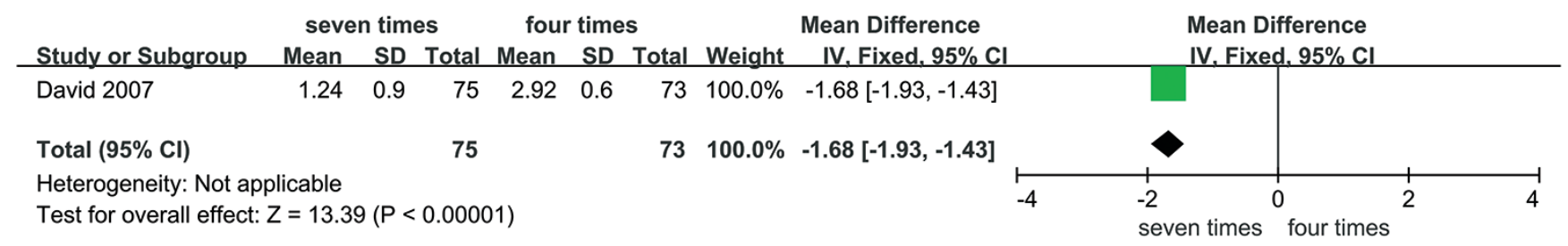

Figure S1 Comparison of the effect of hand washing 7 vs. 4 times per day on the prevention of diarrhea. 